\title{
Averaging in Parametrically Excited Systems - A State Space Formulation
}

\author{
Bastian Pfau $^{1, a}$, Thomas Breunung ${ }^{1}$, Fadi Dohnal ${ }^{2}$, and Richard Markert ${ }^{1}$ \\ 1 Technische Universität Darmstadt, Institute for Applied Dynamics, 64287 Darmstadt, GERMANY \\ 2 Ansaldo Energia Switzerland Ltd, 5401 Baden, SWITZERLAND
}

\begin{abstract}
Parametric excitation can lead to instabilities as well as to an improved stability behavior, depending on whether a parametric resonance or anti-resonance is induced. In order to calculate the stability domains and boundaries, the method of averaging is applied. The problem is reformulated in state space representation, which allows a general handling of the averaging method especially for systems with non-symmetric system matrices. It is highlighted that this approach can enhance the first order approximation significantly. Two example systems are investigated: a generic mechanical system and a flexible rotor in journal bearings with adjustable geometry.
\end{abstract}

\section{Introduction}

Parametrically excited vibrations occur if one or more parameters of a mechanical system, for example stiffness or damping coefficients, are (mostly periodically) varying in time, independently of the system motion. A classic example is the pendulum with periodically moving support leading to the famous MATHIEU equation. A parametrically excited system may exhibit a destabilising parametric resonance, see for example $[3,13]$. In coupled systems of differential equations with time-periodic coefficients not only parametric resonances but also parametric anti-resonances may occur which induce an increased dissipation of vibration energy. The beneficial effect of a parametric antiresonance on self-excited vibration was discovered by Aleš TondL in his pioneering works $[14,15]$. This concept was transferred to general dynamic systems and interpret physically as an energy transfer between the vibration modes of the original system [6], followed by and experimental validation for simple systems including a flexible rotor. A recent summary on this topic can be found in [7]. The stability of such systems can be studied analytically by applying perturbation techniques following the methodologies in $[2,12]$. The present contribution revisits and translates the averaging method of first order, described by

$$
\dot{\hat{\boldsymbol{x}}}=\varepsilon \sum_{k=1}^{K} \frac{1}{T_{k}} \int_{0}^{T_{k}} \boldsymbol{f}^{k}(\hat{\boldsymbol{x}}, t) d t=\varepsilon\langle\boldsymbol{f}(\hat{\boldsymbol{x}}, t)\rangle
$$

to a state-space representation which enables the extension to systems with non-symmetrical matrices and strongly damped vibration modes and applies the methodology to the systems investigated in $[9,11,8]$.

\section{Procedure of Averaging}

The equation of motion of a linear homogeneous mechanical system with $N$ degrees of freedom can be written as

$$
M \ddot{\boldsymbol{q}}+\boldsymbol{B}(t) \dot{\boldsymbol{q}}+\boldsymbol{K}(t) \boldsymbol{q}=\mathbf{0}
$$

with an invertible mass matrix $\boldsymbol{M}$, a speed-proportional matrix $\boldsymbol{B}$ and a deflection-proportional matrix $\boldsymbol{K}$. In general, the matrices $\boldsymbol{B}$ and $\boldsymbol{K}$ are not symmetrical $\left(\boldsymbol{K} \neq \boldsymbol{K}^{T}\right.$ and $\boldsymbol{B} \neq \boldsymbol{B}^{T}$ ), which can be caused by gyroscopic forces, inner damping or journal bearings. Furthermore, $\boldsymbol{B}$ and $\boldsymbol{K}$ alter with the parametric excitation frequency $\Omega_{P}=2 \pi / T_{P}$,

$$
\boldsymbol{K}(t)=\boldsymbol{K}\left(t+T_{P}\right) \quad \text { and } \quad \boldsymbol{B}(t)=\boldsymbol{B}\left(t+T_{P}\right) .
$$

The periodic matrices can be expanded into a FourIER series. Equation (2) then becomes

$$
\begin{aligned}
& \boldsymbol{M} \ddot{\boldsymbol{q}}+ \boldsymbol{B}_{0} \dot{\boldsymbol{q}}+\boldsymbol{K}_{0} \boldsymbol{q}= \\
&-\varepsilon \sum_{k=1}^{K}\left[\left(\boldsymbol{K}^{+} e^{i k \Omega_{P} t}+\boldsymbol{K}^{-} e^{-i k \Omega_{P} t}\right) \boldsymbol{q}\right. \\
&\left.\quad+\left(\boldsymbol{B}^{+} e^{i k \Omega_{P} t}+\boldsymbol{B}^{-} e^{-i k \Omega_{P} t}\right) \dot{\boldsymbol{q}}\right] .
\end{aligned}
$$

In this formulation, constant and varying parts are strictly separated on the left and right hand side. The factor $\varepsilon$ is a small parameter. Formulating equation (4) in state space and transforming the system into modal space using left and right eigenvectors of the underlying system with constant coefficients,

$$
\left[\begin{array}{c}
q \\
\dot{q}
\end{array}\right]=Q\left[\begin{array}{c}
p \\
\dot{p}
\end{array}\right]=Q z
$$

yields

$$
\dot{z}=\Lambda z-\varepsilon \sum_{k=1}^{K}\left(\boldsymbol{P}^{+} e^{i k \Omega_{P} t}+\boldsymbol{P}^{-} e^{-i k \Omega_{P} t}\right) z .
$$

Here, $\Lambda$ is the diagonal matrix of the eigenvalues $\lambda_{n}$ of the underlying system with constant coefficients, $\boldsymbol{z}^{T}=\left[\boldsymbol{p}^{T}, \dot{\boldsymbol{p}}^{T}\right]^{T}$ are the modal coordinates and the matrices $\boldsymbol{P}^{+}$and $\boldsymbol{P}^{-}$arise due to the parametric excitation and couple the modal degrees of freedom. For the $n$-th degree of freedom, equation (6) can be written as

$$
\dot{z}_{n}=\lambda_{n} z_{n}-\varepsilon \sum_{m=1}^{2 N} \sum_{k=1}^{K}\left(P_{n, m}^{+} e^{i k \Omega_{P} t}+P_{n, m}^{-} e^{-i k \Omega_{P} t}\right) z_{m} .
$$

\footnotetext{
a e-mail: pfau@ad.tu-darmstadt.de
} 
Introducing the dimensionless time $\tau=\Omega_{P} t$ and allowing a small detuning $\Delta \Omega_{P}$ of the parametric excitation frequency,

$$
\Omega_{P}=\Omega_{P 0}+\varepsilon \Delta \Omega_{P}+O\left(\varepsilon^{2}\right),
$$

the TAYLOR series expansion of equation (7) for small values of $\varepsilon$ is given by

$$
\begin{aligned}
z_{n}^{\prime}=\bar{\lambda}_{n} z_{n} & -\frac{\varepsilon}{\Omega_{P 0}}\left[\sum _ { m = 1 } ^ { 2 N } \sum _ { k = 1 } ^ { K } \left(P_{n, m}^{+} e^{i k \Omega_{P} t}\right.\right. \\
& \left.\left.+P_{n, m}^{-} e^{-i k \Omega_{P} t}\right) z_{m}+\Delta \Omega_{P} \bar{\lambda}_{n} z_{n}\right]+O\left(\varepsilon^{2}\right) .
\end{aligned}
$$

At this, $\bar{\lambda}_{n}=\lambda_{n} / \Omega_{P 0}$ is a dimensionless eigenvalue and the mark' denotes the derivative with respect to the dimensionless time $\tau$. Making an ansatz of the type

$$
z_{n}=u_{n}(\tau) e^{i \tau \mathfrak{I}\left\{\bar{\lambda}_{n}\right\}},
$$

which is equivalent to the approach of slowly changing phase and amplitude (see e.g. [2]), and rescale the real parts of the eigenvalues,

$$
\mathfrak{R}\left\{\bar{\lambda}_{n}\right\} \mapsto \varepsilon \mathfrak{R}\left\{\bar{\lambda}_{n}\right\}
$$

leads to the standard form and the method of averaging for quasiperiodic systems as outlined in [12] can be applied:

$$
\begin{aligned}
& u_{n}^{\prime}= \varepsilon f(u, t)+O\left(\varepsilon^{2}\right)=-\frac{\varepsilon}{\Omega_{P 0}}\left[\sum _ { m = 1 } ^ { 2 N } \sum _ { k = 1 } ^ { K } \left(P_{n, m}^{+} e^{i k \tau}\right.\right. \\
&\left.+P_{n, m}^{-} e^{-i k \tau}\right) e^{i \tau\left(\mathfrak{J}\left\{\bar{\lambda}_{m}\right\}-\mathfrak{J}\left\{\bar{\lambda}_{n}\right\}\right)} u_{m} \\
&\left.+\left(i \Delta \Omega_{P} \mathfrak{J}\left\{\bar{\lambda}_{n}\right\}-\mathfrak{R}\left\{\bar{\lambda}_{n}\right\} \Omega_{P 0}\right) u_{n}\right]+O\left(\varepsilon^{2}\right) .
\end{aligned}
$$

Averaging the harmonic functions yields always zeros, unless the term becomes resonant. The terms of equation (12) and their resonance condition are given in table 1. Summarizing the last two terms leads to the parametric resonance frequencies at which parametric resonances and anti-resonances can occur,

$$
\Omega_{P 0}=\frac{\mp \mathfrak{I}\left\{\lambda_{n}\right\} \pm \mathfrak{I}\left\{\lambda_{m}\right\}}{k} .
$$

Compared to the standard literature, e. g. [3,13], the imaginary parts of the eigenvalues decide on the parametric resonance frequencies, not the undamped eigenfrequencies.

\begin{tabular}{c|c} 
summand & resonance condition \\
\hline$i \Delta \Omega \mathfrak{J}\left\{\bar{\lambda}_{n}\right\}$ & always \\
$\mathfrak{R}\left\{\bar{\lambda}_{n}\right\} \Omega_{P 0}$ & always \\
$P_{n, m}^{+}$ & $-\mathfrak{J}\left\{\bar{\lambda}_{n}\right\}+\mathfrak{J}\left\{\bar{\lambda}_{m}\right\}+k=0$ \\
$P_{n, m}^{-}$ & $-\mathfrak{J}\left\{\bar{\lambda}_{n}\right\}+\mathfrak{J}\left\{\bar{\lambda}_{m}\right\}-k=0$
\end{tabular}

Table 1. Remaining terms (resonant terms) and their conditions after applying the method of averaging on equation (12)

From now on, higher orders of the FourIER series are neglected, $K=1$. A second order approximation in $\varepsilon$ and a consideration of higher orders in $k$ are discussed in [4].

\section{General case}

If the parametric excitation frequency is not equal to a parametric resonance frequency, averaging of equation (12) yields

$$
\hat{u}_{n}^{\prime}=-\frac{\varepsilon}{\Omega_{P 0}} \sigma_{n} \hat{u}_{n}+O\left(\varepsilon^{2}\right)
$$

with

$$
\sigma_{n}=i \frac{\mathfrak{J}\left\{\lambda_{n}\right\} \Delta \Omega_{P}}{\Omega_{P 0}}-\mathfrak{R}\left\{\lambda_{n}\right\}
$$

for all $n=1 \ldots 2 N$.

\section{Parametric resonances and anti-resonances}

If the parametric excitation frequency is equal to a parametric resonance frequency or in other words, equation (13) is fulfilled for certain values of $n=\tilde{n}$ and $m=\tilde{m}$, averaging of equation (12) yields

\begin{tabular}{|c|c|c|c|c|}
\hline \multicolumn{2}{|c|}{ resonance frequency } & resonance condition & $\bar{n}$ & $\bar{m}$ \\
\hline 1. & - & $2 \mathfrak{J}\left\{\bar{\lambda}_{\bar{n}}\right\} \mp 1=0$ & $\tilde{n}$ & $\tilde{n}+N$ \\
\hline \multirow{2}{*}{2.} & difference & $\mathfrak{J}\left\{\bar{\lambda}_{\bar{n}}\right\}-\mathfrak{J}\left\{\bar{\lambda}_{\bar{m}}\right\} \mp 1=0$ & $\begin{array}{c}\tilde{n} \\
\tilde{m}+N\end{array}$ & $\begin{array}{c}\tilde{m} \\
\tilde{n}+N\end{array}$ \\
\hline & sum & $\mathfrak{I}\left\{\bar{\lambda}_{\bar{n}}\right\}+\mathfrak{I}\left\{\bar{\lambda}_{\bar{m}}\right\} \mp 1=0$ & $\begin{array}{l}\tilde{n} \\
\tilde{m}\end{array}$ & $\begin{array}{l}\tilde{m}+N \\
\tilde{n}+N\end{array}$ \\
\hline
\end{tabular}

$$
\left[\begin{array}{c}
\hat{u}_{\bar{n}}^{\prime} \\
\hat{u}_{\bar{m}}^{\prime}
\end{array}\right]=-\frac{\varepsilon}{\Omega_{P 0}}\left[\begin{array}{cc}
\sigma_{\bar{n}} & P_{\bar{n}, \bar{m}}^{ \pm} \\
P_{\bar{m}, \bar{n}}^{\mp} & \sigma_{\bar{m}}
\end{array}\right]\left[\begin{array}{c}
\hat{u}_{\bar{n}} \\
\hat{u}_{\bar{m}}
\end{array}\right]+O\left(\varepsilon^{2}\right) .
$$

This simplified equation covers the cases for fundamental parametric resonance frequencies (1st kind) and parametric combination resonance frequencies (2nd kind). Table 2 lists the indices for the substitution of $\bar{n}$ and $\bar{m}$ in equation (15). If the resonance condition with the upper sign (column 3 in table 2) is fulfilled, the upper signs in exponent of the counter diagonal elements are valid. Analogous holds for the lower signs.

Table 2. Indices for equation (15) for the modes $\tilde{n}$ and $\tilde{m}$ in dependence of kind and type of the parametric resonance frequency; $+N$ denotes the complex conjugated eigenvalue

Equation (15) is not valid in this simplified form if some eigenvalues have the same imaginary part as $\lambda_{\bar{n}}$ or $\lambda_{\bar{m}}$. This is for example the case if purely real eigenvalues are existing. In this case, averaging of equation (12) leads to a larger matrix which is shown in [4] in more detail.

\section{Stability investigation}

In this section, the calculation of the stability of the averaged System $\hat{\boldsymbol{u}}^{\prime}=\boldsymbol{A} \hat{\boldsymbol{u}}$ (equation (14) respectively (15)) under the neglection of higher orders in $\varepsilon$ is presented. If the parametric resonance frequencies are not close to each other, an interaction between them can be ruled out. In this case, it is sufficient to consider one parametric resonance frequency for the calculation of each stability domain, which is the common method in literature [12]. If two or more stability domains overlap then the isolated treatment of only one parametric resonance frequency is strictly no longer valid. The present investigation establishes an empiric approach which takes all involved parametric resonances into account.

\subsection{No overlapping of parametric resonances}

This subsection is, analogous to section 2, divided into a general case and into the case of parametric resonances and anti-resonances. 


\section{General case}

Investigating the stability of equilibria of equation (14) yields one stability condition,

$$
\mathfrak{R}\left\{\lambda_{n}\right\}<0,
$$

for all $n=1 \ldots 2 N$. This condition is also to find in every good book on structural dynamics [10] or systems theory.

\section{Parametric resonances and anti-resonances}

Investigating the stability of equilibria of equation (15) using the criteria from Routh and HuRwitz for complex matrices (see [1]) yields the stability conditions

$$
\mathfrak{R}\left\{\lambda_{\bar{n}}\right\}+\mathfrak{R}\left\{\lambda_{\bar{m}}\right\}<0
$$

and

$$
\Delta \Omega_{P}^{2}+2 a_{1} \Delta \Omega_{P}+a_{0}>0 .
$$

For the remaining modal degrees of freedom, $n \neq \tilde{n}, \tilde{m}$, $\tilde{n}+N, \tilde{m}+N$, equation (16) has to be fulfilled for stability. Setting equation (18) equal to zero, critical values for $\Delta \Omega_{P}$ are calculated to

$$
\Delta \Omega_{P, c}^{ \pm}=-a_{1} \pm \sqrt{a_{1}^{2}-a_{0}}=\Delta \Omega_{P, s} \pm \Delta \Omega_{P, w} .
$$

Herein, $\Delta \Omega_{P, s}$ defines the distance between the parametric resonance frequency and the skeleton line of the stability domain, also denoted as frequency shift (see e.g. [5]), and $\Delta \Omega_{P, w}$ is the width of the stability domain. The parameters $a_{1}$ and $a_{0}$ are given by

$$
2 a_{1}=C_{1} C_{2}\left(\mathfrak{R}\left\{\lambda_{\bar{m}}\right\}-\mathfrak{R}\left\{\lambda_{\bar{n}}\right\}\right)
$$

and

$$
\begin{aligned}
& a_{0}=\frac{C_{1} \Omega_{P 0}}{\mathfrak{I}\left\{\lambda_{\bar{m}}\right\}-\mathfrak{J}\left\{\lambda_{\bar{n}}\right\}}\left[-C_{2}^{2}+\left(\mathfrak{R}\left\{\lambda_{\bar{n}}\right\}+\mathfrak{R}\left\{\lambda_{\bar{m}}\right\}\right)^{2}\right. \\
& \left.\cdot\left(\mathfrak{I}\left\{P_{\bar{n} \bar{m}}^{ \pm}\right\} \mathfrak{J}\left\{P_{\bar{m} \bar{n}}^{\mp}\right\}-\mathfrak{R}\left\{P_{\bar{n} \bar{m}}^{ \pm}\right\} \mathfrak{R}\left\{P_{\bar{m} \bar{n}}^{\mp}\right\}+\mathfrak{R}\left\{\lambda_{\bar{n}}\right\} \mathfrak{R}\left\{\lambda_{\bar{m}}\right\}\right)\right]
\end{aligned}
$$

with

$$
\begin{aligned}
& C_{1}=\Omega_{P 0}\left[\mathfrak{R}\left\{\lambda_{\bar{n}}\right\} \mathfrak{R}\left\{\lambda_{\bar{m}}\right\}\left(\mathfrak{J}\left\{\lambda_{\bar{m}}\right\}-\mathfrak{I}\left\{\lambda_{\bar{n}}\right\}\right)\right]^{-1}, \\
& C_{2}=\mathfrak{R}\left\{P_{\overline{\bar{n}} \bar{m}}^{ \pm}\right\} \mathfrak{I}\left\{P_{\bar{m} \bar{n}}^{\mp}\right\}+\mathfrak{R}\left\{P_{\bar{m} \bar{n}}^{\mp}\right\} \mathfrak{I}\left\{P_{\overline{\bar{n}} \bar{m}}^{ \pm}\right\} .
\end{aligned}
$$

This defines a domain of stability (respectively instability) which is given by

$$
\begin{aligned}
\Omega_{P 0}+\varepsilon\left(\Delta \Omega_{P, s}\right. & \left.-\Delta \Omega_{P, w}\right)<\Omega_{P} \\
& <\Omega_{P 0}+\varepsilon\left(\Delta \Omega_{P, s}+\Delta \Omega_{P, w}\right) .
\end{aligned}
$$

The given solution is only valid if the imaginary parts of all other eigenvalues $\lambda_{n}(n \neq \bar{n}, \bar{m})$ are not equal to $\mathfrak{J}\left\{\lambda_{n}\right\}$ or $\mathfrak{J}\left\{\lambda_{m}\right\}$. For these cases an analytical solution is not found yet and have to be treated using numerical calculations.

\subsection{Overlapping of parametric resonances}

If parametric resonances and anti-resonances are close to each other, the way described in section 3.1 may lead to contradictory results, due to the isolated treatment of only one parametric resonance frequency. An empirical approach is proposed here based on the knowledge that the eigenvalues of the averaged system $\hat{\boldsymbol{u}}^{\prime}=\boldsymbol{A} \hat{\boldsymbol{u}}$ at parametric excitation (equations (15)) differ from the eigenvalues $\lambda$ of the system with constant coefficients (see [6]). The interaction of $R$ involved parametric resonance frequencies $\Omega_{P 0}^{r}$ $(r=1 \ldots R)$ is investigated. The eigenvalue of the averaged system $\hat{\boldsymbol{u}}^{\prime}=\boldsymbol{A} \hat{\boldsymbol{u}}$ for the $n$-th modal degree of freedom and the $r$-th parametric resonance frequency $\Omega_{P 0}^{r}$ (see table 2$)$ is calculated to

$$
\rho_{n}^{r}=\left\{\begin{array}{ll}
\operatorname{eig}\left(\boldsymbol{A}\left(\Omega_{P 0}^{r}\right)\right) & \text { if } n=\bar{n}, \bar{m} \\
\lambda_{n} & \text { if } n \neq \bar{n}, \bar{m}
\end{array} .\right.
$$

If the upper condition in equation (24) is fulfilled, the corresponding eigenvalue depends on the parametric excitation frequency, $\rho_{n}^{r}=\rho_{n}^{r}\left(\Omega_{P}\right)$. The real part of the parameter

$$
\Delta \rho_{n}^{r}=\rho_{n}^{r}-\lambda_{n}
$$

can be interpret as a measure of additional damping (negative or positive) which is caused by the $r$-th parametric resonance frequency $\Omega_{P 0}^{r}$. Summing these parameters over all $r$ yields

$$
\tilde{\rho}_{n}=\lambda_{n}+\sum_{r=R}^{R} \Delta \rho_{n}^{r} .
$$

The real part of the parameter $\tilde{\rho}_{n}$ is used to evaluate the stability without a contradiction. For stability the real parts of $\tilde{\rho}_{n}$ need to be negative for all $n=1 \ldots 2 N$.

\section{Examples}

The presented equations are applied on two exemplary mechanical systems which have been studied in literature.

\subsection{Generic mechanical system}

First of all, a generic system with two degrees of freedoms from [9] is investigated, it is shown in figure 1. The system consists of two bodies that are connected by a spring with a time-periodic stiffness, $k_{2}(t)=\hat{k}_{2}\left(1+\varepsilon \cos \Omega_{P} t\right)$. The damping constant $b_{1}$ is negative which has an destabilizing effect on the system. The physical properties are listed in [9].

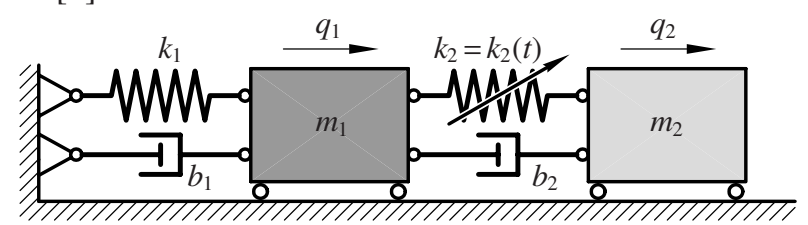

Fig. 1. Two degree of freedom system with stiffness excitation

Figure 2 shows a stability map in dependence of the parametric excitation frequency $\Omega_{P} / \sqrt{k_{1} / m_{1}}$ and the variable system parameter $Q^{2}=k_{2} m_{1} /\left(k_{1} m_{2}\right)$. The gray and white areas depict stable and unstable areas, they are computed by numerical calculation using FLOQUET theory and are used for comparison purposes. The black and and gray lines are the stability boundaries calculated with the method of averaging of the first order presented in [9] and the state space representation in equation (12). The trivial equilibrium position of the system without parametric excitation is stable for $0.934<Q<0.964$. An additional stability domain occurs due to a parametric anti-resonance at the parametric resonance frequency $\left|\mathfrak{J}\left\{\lambda_{2}\right\}-\mathfrak{J}\left\{\lambda_{1}\right\}\right|$. Figure 2 visualizes that the black lines and the stability boundaries from FLoQUET theory match very well. Comparing the results from the different approaches of the averaging method show that the formulation in state space enhances the first order approximation significantly and shows the same accuracy as the higher order approximation in [9]. 


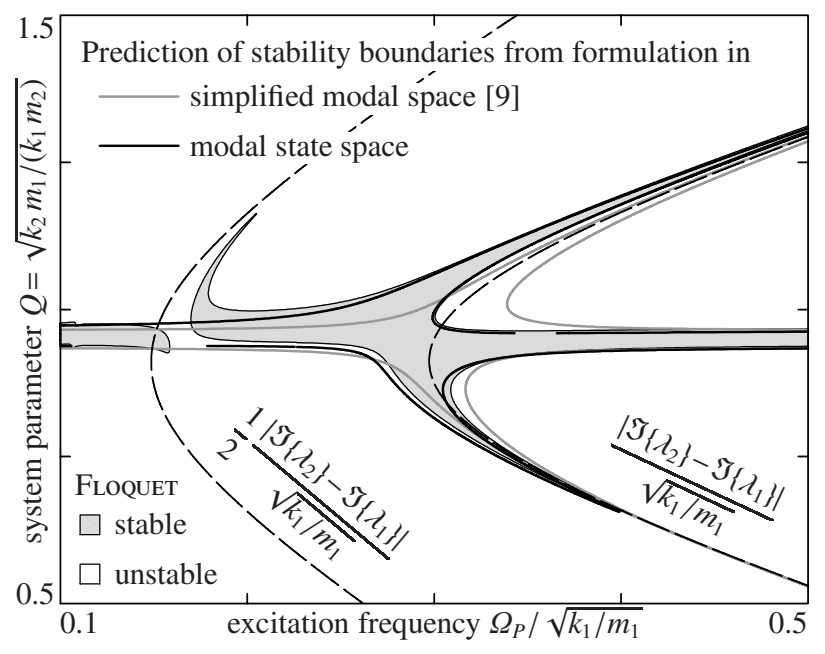

Fig. 2. Stability map for the generic system $(\varepsilon=0.3)$

\subsection{Flexible rotor in journal bearings}

The second example is a flexible rotor in journal bearings with adjustable geometry, the problem is described in detail in $[11,8]$. The aim of the proposed journal bearing is a suppression of an instability induced by the bearing (oil whip) which has to be avoided in any case. Figure 3 shows an example of a stability map. The system without parametric excitation is stable up to a rotational speed of $\Omega / \omega_{0} \approx 2.4$ which limits the permissible speed range. By exploiting a parametric anti-resonance $\left(\left|\mathfrak{I}\left\{\lambda_{3}\right\} \mathfrak{I}\left\{\lambda_{1}\right\}\right| / \omega_{0}\right)$, the operation speed range is increased significantly.

In this example, treating of one single parametric resonance frequency (chapter 3.1) causes a relatively large deviation in comparison to the results from FloqueT theory. The reason for this is the interaction of several parametric resonance frequencies, especially a coupling with the strongly damped eigenvalue $\left|\lambda_{7}\right|$.Taking them into account and applying the empirical method described in 3.2 (equation (26)) yields a better approximation which allows for engineering design optimisations.

\section{Conclusions}

In this paper, the method of averaging was applied on linear parametrically excited systems. It was shown that the formulation in state space implicates several advantages: an improved approximation as well as an enhanced handling of the averaging method. An experimental validation for the suppression of bearing induced instabilities using parametric anti-resonances is in progress.

\section{Acknowledgements}

This work is dedicated to Aleš TondL $(* 1925, \dagger 2016)$. He was an excellent scientist in the field of vibrations and rotor dynamics. The present work would not exist without his discovery of the beneficial effect of parametric antiresonance.

\section{References}

1. H. Bilharz. Bemerkung zu einem Satze von Hurwitz. Z. angew. Math. Mech., 24(2):77-82, 1944.

2. N. Boguliubov and Y. A. Mitropolskij. Asymptotische Methoden in der Theorie der nichtlinearen Schwingungen. Akademie Verlag Berlin, 1965.

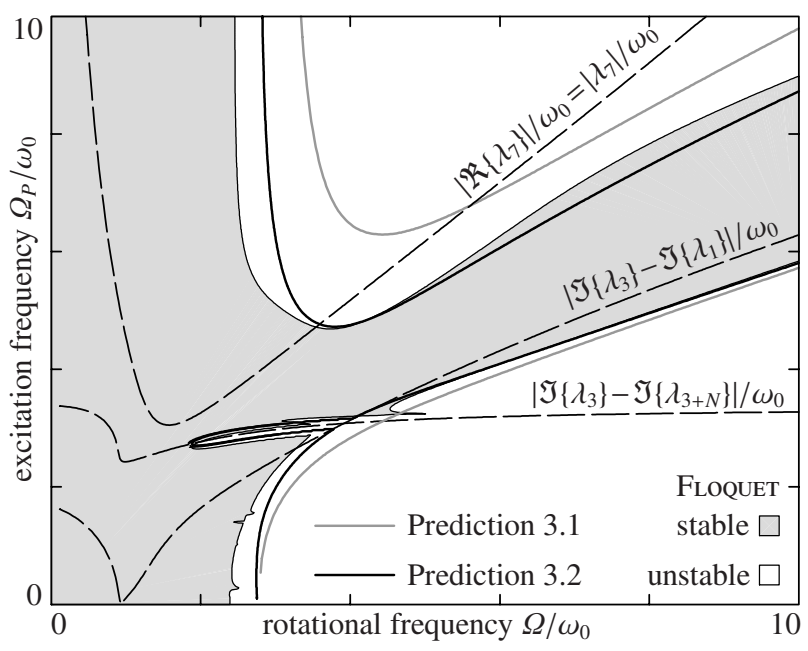

Fig. 3. Stability map for a rotor system in journal bearings with adjustable geometry (see also [11] and [8])

3. V. Bolotin. The Dynamic Stability of Elastic Systems. Holden-Day, San Francisco, California, 1964.

4. T. Breunung. Semianalytische Berechnungen von Stabilitätskarten eines gleitgelagerten Rotorsystems mit Parametererregung. Masterthesis, Technische Universität Darmstadt, 2016.

5. F. Dohnal. Suppressing self-excited vibrations by synchronous and time-periodic stiffness and damping variation. Journal of Sound and Vibration, 306:136152, 2007.

6. F. Dohnal. Damping by Parametric Stiffness Excitation: Resonance and Anti-Resonance. Journal of $\mathrm{Vi}$ bration and Control, 14(5):669-688, 2008.

7. F. Dohnal. A contribution to the mitigation of transient vibrations, Parametric anti-resonance: theory, experiment and interpretation. habilitation thesis, Technische Universität Darmstadt, 2012.

8. F. Dohnal, B. Pfau, and A. Chasalevris. Analytical predictions of a flexible rotor in journal bearings with adjustable geometry to suppress bearing induced instabilities. In Proceedings of the 13th Int. Conf. on Dynamical Systems - Theory and Applications, Łódź, Poland, December 2015.

9. F. Dohnal and F. Verhulst. Averaging in vibration suppression by parametric stiffness excitation. Nonlinear Dynamics, 54:231-248, 2008.

10. R. Gasch, K. Knothe, and R. Liebich. Strukturdynamik - Diskrete Systeme und Kontinua. Springer, 2012.

11. B. Pfau, M. Rieken, and R. Markert. Numerische Untersuchungen eines verstellbaren Gleitlagers zur Unterdrückung von Instabilitäten mittels ParameterAntiresonanzen. In First IFToMM D-A-CH Conference, Dortmund, Germany, 2015.

12. J. A. Sanders, F. Verhulst, and J. Murdock. Averaging Methods in Nonlinear Dynamical Systems. Spr., 2007.

13. G. Schmidt. Parametererregte Schwingungen. Deutscher Verlag der Wissenschaften, Berlin, 1975.

14. A. Tondl. On the interaction between self-excited and parametric vibrations. Monographs and Memoranda No. 25, National Research Institute for Machine Design, Běchovice, 1978.

15. A. Tondl. To the Problem of Quenching Self-Excited Vibrations. Acta Techn. CSAV, 43:109-116, 1998. 